\title{
In-Vitro Biological Experiment for New Pharmacology Compounds at High-Altitude-Area, Taif, KSA
}

\author{
Sherifa Mostafa M. Sabra* \\ Senior Const., Asst. Prof., Dr., Specialty Microbiology \\ Micro. Br., Biology Dept., Sci. College, Taif University, KSA \\ Serology Unit and Bacterial Strains Bank, [AHRI], Dokki, [ARC], Giza, Egypt.
}

*Corresponding Author: Sherifa Mostafa M. Sabra, Serology Unit and Bacterial Strains Bank, [AHRI], Dokki, [ARC], Giza, Egypt.

\begin{abstract}
This paper was discharged for "In-vitro biological experiment for new pharmacology compounds at high-altitude-area (HAA), Taif, KSA". The mean of antimicrobial effects on Staphylococcous aureus (SA), as antibacterial effect were at $(1,3,5$ and $7 \mathrm{hr})$. The mean antimicrobial were in descendant order by antibacterial as followed (K1, K2, k3, K4, K5 and K6) were 100\% at 7hr. Nevertheless, (K3 and K6) were $100 \%$ since $5 \mathrm{hr}$. The newly synthesized compounds inhibitory concentration presented high important activity against $S A$. The mean of antimicrobial effects on Escherichia coli (EC), as antibacterial more effective (K1, $\mathrm{K} 2, \mathrm{~K} 3$ and $\mathrm{K5}$ ) were $100 \%$ at $7 \mathrm{hr}$, then (K4 and K5) were (95 and 90\%). The mean antibacterial during the exposure hrs and the averages were in descendent command by antibacterial fraction. The inhibitory concentration presented by using pharmacology compounds high important activity against EC. The mean of antimicrobial effects on Candida albicans (CA), the effect as antifungal were included (1, 3, 5 and $7 h r)$. The means were in descendant order by the fraction of antifungal, the more effective were (K2, K3 and K6) were $100 \%$ at $7 \mathrm{hr}$, and then (K1, K4 and K5) were (90, 95 and 85\%). The effective was clear with pharmacology compounds. This present applied work concluded pharmacology compounds had effective more powerful than antimicrobial agents did. As well, it could be institution of antimicrobial agents for "Health-program" (HP) at HAA. Using new pharmacology compounds instead of chemical antimicrobial substances to protect human body from side effects and could improve HP at HAA.
\end{abstract}

Keywords: In-vitro Biological Experiment, Pharmacology Compounds, High-altitude-area, Staphylococcous aureus, Escherichia coli, Candida albicans.

ABBREVIATION
\begin{tabular}{|c|c|c|}
\hline Candida albicans: $(C A)$ & Health-program: $(H P)$ & Staphylococcous aureus: $($ SA) \\
\hline Escherichia coli: $($ EC) & High-altitude-area: $(H A A)$ & \\
\hline
\end{tabular}

\section{INTRODUCTION}

Microbes as bacteria and fungi had confrontation in contradiction of antimicrobial agents, it is vital to project antimicrobial mediators less toxic and strong. Chemists substitution had manufactured actual, examples was midazoles had numerous of pharmacology belongings as antimicrobial [1]. Midazole and its derivatives production had a part in antimicrobial; its derivatives had recent manufactured and dah same characters [2]. It had before created midazole byproducts and had antimicrobial effects [34]. Midazole derivatives possess varied biochemical constructions might useful expansion of antimicrobial effects. The progress of well-organized midazole romped a main in current biological mixture [5]. The negligible inhibitory attention for manufactured mixes exhibited in height significant antimicrobials [6]. Sequences of derivatives were killed $S A, E C$ and $C A$ [7].

The aim of this work was for detection of new pharmacology compounds by In-vitro biological experiment as pathogenic microbes at HAA, Taif, KSA. That for proven the ability of new pharmacology compounds in kill drug resistant microbes to decrease the side effects of health HP at HAA. 


\section{Materials AND Methods}

- New pharmacology compounds from midazole and derivatives were collected [8].

- Microbial isolates collected, microbial suspension made as McFarland [9].

- New pharmacology compounds practical mixed with microbial suspension. The mixtures were incubated at $37^{\circ} \mathrm{C}$ for $7 \mathrm{hr}$. Colony growth were examined every $(1,3,5$ and $7 \mathrm{hr})$, by nutrient agar plate, were incubated at $37^{\circ} \mathrm{C}$ for $48 \mathrm{hr}$ [10].

- Microbial death percent calculated used an Equation: [(Colony No. / 300 X 100) -100] [11].

- Data examination: "Simple Basic Excel Formulas" was persistent for conduct the results [12].

3. RESULTS AND DisCUSSION

Table1 and graph1. The mean of antimicrobial effects on *SA

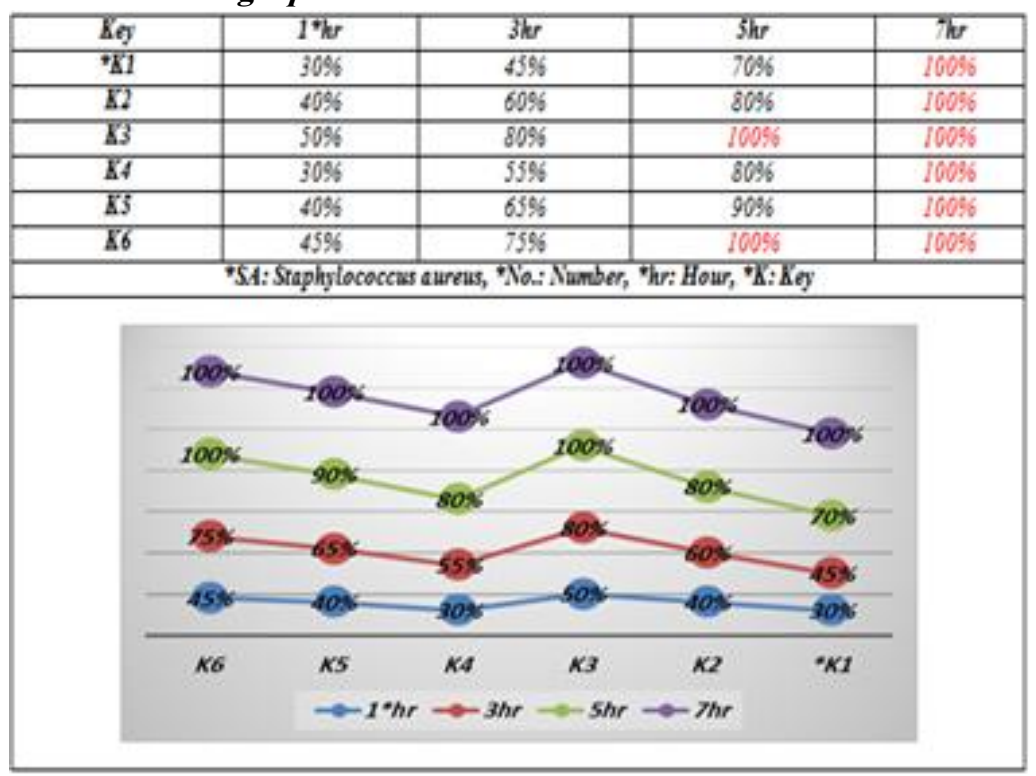

Table 1 and graph 1 revealed the mean of antimicrobial effects on $S A$, which revealed the effect as antibacterial and were in $(1,3,5$ and $7 \mathrm{hr})$. The mean antimicrobial were in descendant order by antibacterial as followed (K1, K2, k3, k4, K5 and K6) were 100\% at 7hr. Nevertheless, (K3 and K6) were $100 \%$ since $5 \mathrm{hr}$. The negligible inhibitory concentration for newly synthesized compounds presented high important activity against $S A$ [6-7].

Table 2 and graph2. The mean of antimicrobial effects on $* \mathrm{EC}$

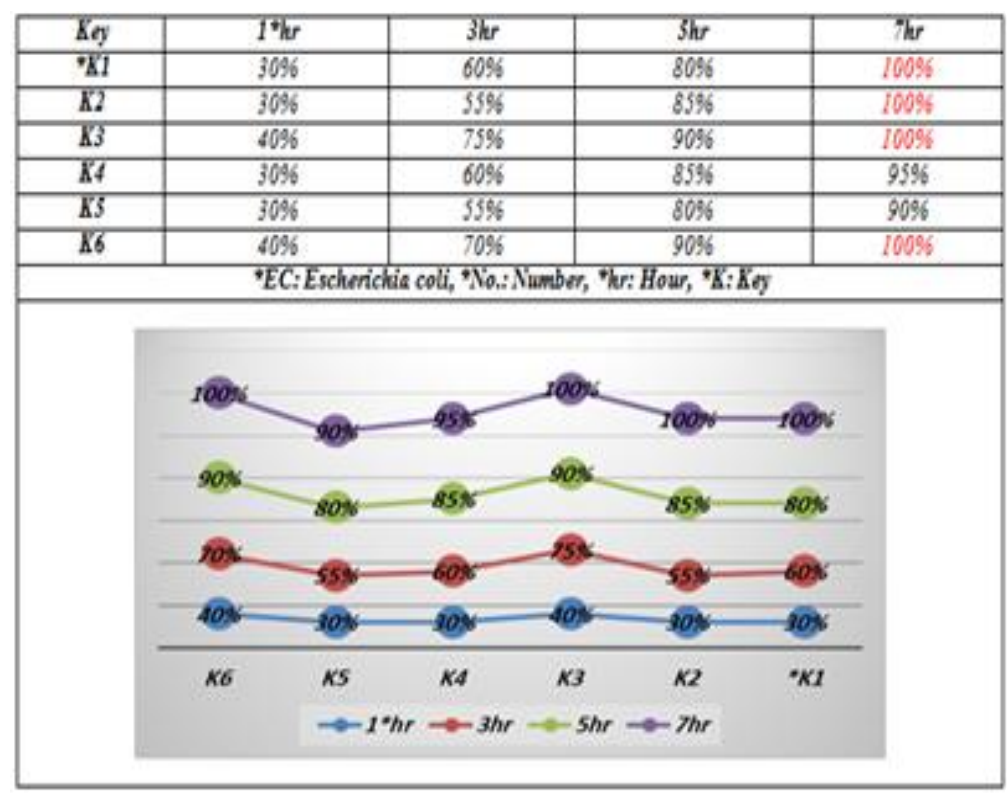


Table 2 and graph 2 revealed the mean of antimicrobial effects on $E C$, that revealed the effect as antibacterial more effective for (K1, K2, K3 and K5) were $100 \%$ at $7 \mathrm{hr}$, then (K4 and K5) were (95 and $90 \%$ ). The mean antibacterial during the exposure hrs and the averages were in descendent command by antibacterial fraction. The inhibitory concentration presented by using pharmacology compounds high important activity against $E C$ [6-7].

Table 3 and graph 3. The mean of antimicrobial effects on $* C A$

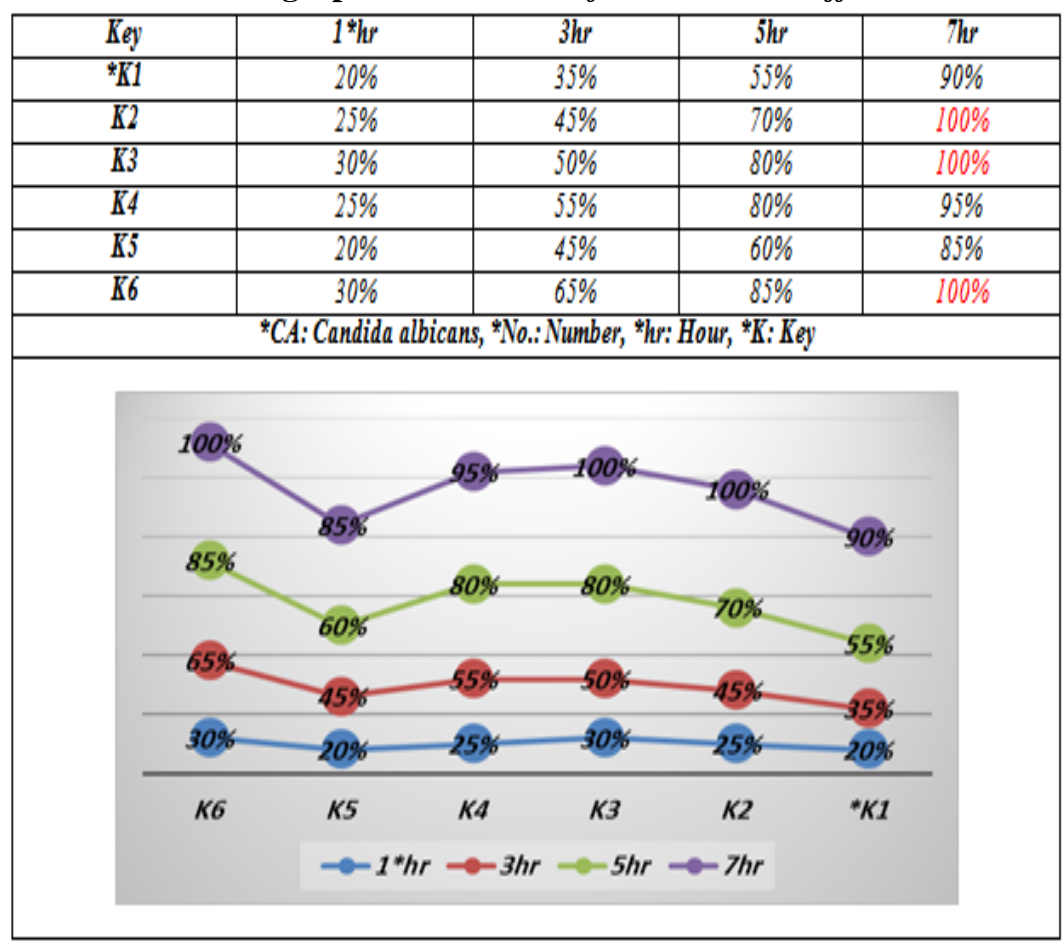

Table 3 and graph 3 revealed the mean of antimicrobial effects on $C A$, the effect as antifungal and were included (1, 3, 5 and $7 \mathrm{hr})$. The mean effects were intended and the means were in descendant order by the fraction of the antifungal, the more effective were (K2, K3and K6) were in $100 \%$ at $7 \mathrm{hr}$, and then for (K1, K4 and K5) were (90, 95 and 85\%). The effective was clear with pharmacology compounds [6-7].

\section{CONCLUSION}

This present applied work concluded the pharmacology compounds have effective more powerful than as antimicrobial agents. As well, it could be institution in place of antimicrobial agents for HP at HAA.

\section{RECOMMENDATION}

Using pharmacology compounds instead of antimicrobial to protect human body from side effects and could improve HP at HAA.

\section{ACKNOWLEDGMENT}

Many thanks were sent for all persons were helped in produce this paper.

\section{REFERENCES}

[1] Sekar, U., Padalkar, B., Gupta, V., Pharangare, K., Patil, V. and Umape, P., Synthesis and antimicrobial activity of novel 2-substituted benzimidazole, benzoxazole and benzothiazole derivatives. Arab. J. Chem., 2016; 9:S1125-S1130.

[2] Alaqeel, S., Synthetic approaches to benzimidazoles from ophenylenediamine: A literature review. J. Saudi Chem. Soc., 2017; 21:229-237.

[3] Desai, N., Joshi, V., Rajpara, K., Vaghani, H., Satodiya, H. and Bhatt, K., Synthesis and evaluation of Nsubstituted thiazolidine-2,4-dione containing pyrazole as a potent antimicrobial agents. Anti-infective agents, 2014a; 12:85-94.

[4] Desai, N., Shihory, N. and Kotadiya, G., Facile synthesis of benzimidazole bearing 2-pyridone derivatives as potential antimicrobial agents. Chin. Chem. Lett., 2014b; 25:305-307. 
[5] Padmaja, A., Rajasekhar, C., Muralikrishna, A. and Padmavathi, V., Synthesis and antioxidant activity of oxazolyl/thiazolyl sulfonyl methyl pyrazoles and isoxazoles. Eur. J. Med. Chem., 2011; 6:5034-5038.

[6] Haggam, R., El-Sayed, H., Said, S., Ahmed, M., Moustafa, A and Abd-El-Noora, R., Oglycosylation/alkylation and antimicrobial activity of 4, 6-diaryl-2-oxonicotinonitrile derivatives. J. Heterocyclic. Chem., 2017; 54: 375-383

[7] Desai, N., Darshan, P. and Darshita, V., Synthesis and antimicrobial activity of some heterocyclic compounds bearing benzimidazole and pyrazoline motifs. Med. Chem. Res., 2017; DOI 10.1007/s00044017-2040-5.

[8] Esmaeili, N., Ebrahimzadeh, H., Abdi, K. and Safarian, S., Determination of some phenolic compounds in Crocus sativus L. corms and its antioxidant activities study. Pharmacogn. Mag., 2011; 7(25):74-80.

[9] CLSI (Clinical and Laboratory Standards Institute) Performance for antimicrobial disk susceptibility tests; approved standard. 11. Wayne (PA), USA: Clinical and Laboratory Standards Institute; 2012.

[10] https://archive.org/stream/manualofmicrobio00amer/manualofmicrobio00amer_djvu.txt.2019

[11] https://arxiv.org/pdf/1701.05870.pdf.2019

[12] https://people.umass.edu/evagold/excel.html2019

Citation: Sherifa Mostafa M. Sabra, "In-Vitro Biological Experiment for New Pharmacology Compounds at High-Altitude-Area, Taif, KSA”. International Journal of Research Studies in Biosciences (IJRSB). 7(10), pp. 22-25. DOI: http://dx.doi.org/10.20431/2349-4050.0710003

Copyright: () 2019 Authors this is an open-access article distributed under the terms of the Creative Commons Attribution License, which permits unrestricted use, distribution, and reproduction in any medium, provided the original author and source are credited. 